\title{
Pulmonary Hypertension in COPD
}

\author{
Matthias Held MD and Berthold H Jany MD PhD
}

\begin{abstract}
Pulmonary hypertension (PH) is a common consequence of COPD. It has been speculated that patients showing serious $\mathbf{P H}$ and vascular remodeling without severe airway obstruction might benefit from vasoactive treatment. There is no approved drug available for COPD-induced PH. Most trials assessing the efficacy of vasoactive drugs in PH have had a follow-up of 12-16 weeks. We report on 4 subjects with COPD and PH. Pulmonary arterial hypertension associated diseases and pulmonary embolism were ruled out. $\mathrm{PH}$ persisted despite optimized treatment of underlying COPD and comorbidities, so bosentan was started in all 4 subjects. With bosentan the mean pulmonary artery pressure improved. The average gains in 6-min walk distance at 2-3 months and 8-9 months were $36 \mathrm{~m}$ and $145 \mathrm{~m}$, respectively. The maximum gains in 6-min walk distance of the individual subjects were at the 9th, 13th, and 18th month. Oxygenation was stable, and no side effects were observed. We suggest from this experience that in clinical trials of PH in COPD, a follow-up of 16 weeks might cause underestimation of the treatment effects. Key words: COPD; pulmonary hypertension; hemodynamics; clinical trials; treatment; vasoactive drugs; follow-up. [Respir Care 2013;58(8):e86-e91. (C) 2013 Daedalus Enterprises]
\end{abstract}

\section{Introduction}

Pulmonary hypertension $(\mathrm{PH})$ is a common consequence of COPD, although the actual overall prevalence of $\mathrm{PH}$ in COPD remains unclear. ${ }^{1}$ Among COPD patients who were to undergo lung-volume-reduction surgery or lung transplantation, mild $\mathrm{PH}$ was observed in $50.2 \%$, moderate $\mathrm{PH}$ in $9.8 \%$, and severe $\mathrm{PH}$ in $3.7 \% .^{2}$ While in most COPD patients who develop $\mathrm{PH}$, pulmonary arterial pressure is only mildly or moderately elevated, ${ }^{3} \mathrm{PH}$ has an independent prognostic impact on survival. ${ }^{3,4}$ Even in a range $(>18 \mathrm{~mm} \mathrm{Hg}$ ) that does not represent $\mathrm{PH}$ as defined by a

The authors are affiliated with the Department of Internal Medicine, Medical Mission Hospital, Julius Maximilian Universität Würzburg, Würzburg, Germany.

Dr Held has disclosed relationships with Actelion, Bayer Healthcare, Boehringer Ingelheim, GlaxoSmithKline, Lilly, Novartis, Nycomed, Pfizer, Roche, and Servier. Dr Jany has disclosed relationships with Actelion, Bayer Healthcare, Boehringer Ingelheim, MSD, and Nycomed.

Correspondence: Matthias Held MD, Department of Internal Medicine, Medical Mission Hospital, Julius Maximilian Universität Würzburg, Salvatorstraße 7, 97064 Würzburg, Germany. E-mail: Matthias. held@missioklinik.de.

DOI: $10.4187 /$ respcare. 02058 mean pulmonary artery pressure of $25 \mathrm{~mm} \mathrm{Hg}$, the rate of hospital admission due to COPD exacerbation is higher than in patients with a mean pulmonary artery pressure $<18 \mathrm{~mm} \mathrm{Hg.}{ }^{3}$ Pulmonary artery pressure is a stronger prognostic factor in patients with COPD than is $\mathrm{FEV}_{1}$, hypoxemia, or hypercapnia. ${ }^{5}$

Although it has been proposed that PH develops due to loss of capillaries in emphysema and chronic hypoxemia, elevated pulmonary artery pressure was not correlated with oxygenation in patients with severe emphysema. Rather, it was associated with elevated pulmonary wedge pressure, reflecting left diastolic dysfunction, despite preserved systolic ventricular function. ${ }^{6}$

A subgroup of subjects with severe PH but only moderate ventilatory disturbance was identified ${ }^{2}$ and classified as "out of proportion PH." 4 Aberrant vascular remodeling induced by tobacco smoke was found, and a correlation with small airway disease and emphysema was established. ${ }^{7}$ It is still unclear whether severe PH in these subjects is the result of COPD or an independently coexisting idiopathic pulmonary arterial hypertension. ${ }^{4}$

In PH occurring due to respiratory disorders and hypoxemia, treatment of the underlying disease and oxygen administration has been recommended. ${ }^{8,9}$ Further evaluation is advised only if the PH is severe. ${ }^{9}$ It was speculated that COPD patients with severe PH but only mild to moderate 
Table. Baseline Characteristics of the 4 Subjects

\begin{tabular}{|c|c|c|c|c|}
\hline & \multicolumn{4}{|c|}{ Subject Number } \\
\hline & 1 & 2 & 3 & 4 \\
\hline Sex & M & $\mathrm{F}$ & M & M \\
\hline Age, y & 78 & 73 & 74 & 75 \\
\hline Body mass index, $\mathrm{kg} / \mathrm{m}^{2}$ & 31.6 & 30.3 & 31.1 & 25.8 \\
\hline $\mathrm{FEV}_{1} / \mathrm{FVC}, \%$ & 45 & 37 & 55 & 50 \\
\hline $\mathrm{FEV}_{1}, \mathrm{~L}$ & 1.13 & 0.65 & 1.42 & 2.27 \\
\hline $\mathrm{FEV}_{1}, \%$ predicted & 39 & 40 & 52 & 63 \\
\hline $\mathrm{FVC}, \mathrm{L}$ & 2.46 & 1.73 & 2.55 & 4.51 \\
\hline $\mathrm{FVC}, \%$ predicted & 61 & 88 & 69 & 90 \\
\hline Intrathoracic gas volume, $\mathrm{L}$ & 4.35 & 3.43 & 3.21 & 5.69 \\
\hline Intrathoracic gas volume, $\%$ predicted & 115 & 137 & 91 & 138 \\
\hline Diffusion capacity, $\%$ predicted & 79 & 43 & 26 & 61 \\
\hline 6-min walk distance, $\mathrm{m}$ & 240 & 165 & 135 & 400 \\
\hline World Health Organization functional class & 3 & 3 & 3 & 3 \\
\hline Mean pulmonary artery pressure at rest, $\mathrm{mm} \mathrm{Hg}$ & 44 & 38 & 50 & 38 \\
\hline Pulmonary artery wedge pressure, $\mathrm{mm} \mathrm{Hg}$ & 15 & 13 & 22 & 15 \\
\hline Transpulmonary pressure difference, $\mathrm{mm} \mathrm{Hg}$ & 26 & 25 & 28 & 23 \\
\hline Pulmonary vascular resistance, $\mathrm{dyn} / \mathrm{s} / \mathrm{cm}$ & 326 & 400 & 476 & 526 \\
\hline Cardiac index, $\mathrm{L} / \mathrm{min} / \mathrm{m}^{2}$ & 3.2 & 2.7 & 2.3 & 1.7 \\
\hline Mean pulmonary artery pressure after iloprost & 28 & Not measured & No change & Not measured \\
\hline
\end{tabular}

ventilatory impairment might benefit from vasoactive medication. ${ }^{2}$ However, the reported effects of vasoactive drugs have been inconsistent ${ }^{10-13}$ and reliable corroborating data are lacking.

\section{Case Reports}

We report on 4 subjects with COPD and PH. In all 4 subjects we did a complete staging of COPD, including body plethysmography, analysis of capillary blood samples for oxygen and carbon dioxide, and high-resolution computed tomography of the lungs. Diagnostic workup for PH was performed according to the guidelines. ${ }^{8}{ }^{8}$ Pulmonary embolism was ruled out by computed tomography and ventilation-perfusion scans. Anorexigen use, congenital heart disease, porto-pulmonary hypertension, collagen vascular disease, human immunodeficiency virus infection, and chronic hemolysis were ruled out. There was no family history of pulmonary arterial hypertension.

The characteristics of the 4 subjects were as follows:

Subject 1: COPD, central sleep related breathing disorders, atrial fibrillation, coronary artery disease

Subject 2: COPD, emphysema, hypoxemia, oxygen administration

Subject 3: COPD, atrial fibrillation, coronary artery disease, prosthetic aortic valve

Subject 4: COPD, atrial fibrillation, coronary artery disease
Treatment of COPD and other accompanying medical conditions were optimized as follows.

Subject 1: Due to central sleep apnea, treatment with CPAP and night-time oxygen supplementation was started, and anti-obstructive treatment of COPD was intensified. The subject was treated with tiotropium bromide and formoterol. Additionally, digitoxin was given instead of a $\beta$-blocker. Rehospitalization for baseline right heart catheter and 6-min walk test was done 3 months later.

Subject 2: As an anti-obstructive treatment the subject received tiotropium bromide and formoterol/budesonide. The treatment was not changed. The subject was admitted for a second-look evaluation and baseline right heart catheter and 6-min walk test 4 weeks later.

Subject 3: Long-term oxygen treatment had been initiated 5 months before baseline right heart catheter. The current treatment with tiotropium bromide and formoterol/ budesonide was continued.

Subject 4: Coronary angioplasty was performed, and tiotropium bromide was started 4 weeks before baseline right heart catheter.

To evaluate the effects of these interventions, all the subjects were admitted at our hospital for a second look 1-5 months later. Their examinations did not show any important improvement of PH. After excluding COPD exacerbation by evaluating dyspnea, cough, and sputum according to the Global Initiative for Chronic Obstructive Lung Disease (GOLD) statement ${ }^{14}$ and performing base- 
line right heart catheterization, 6-min walk test, and cardiopulmonary exercise test, treatment with bosentan was started.

The Table shows the baseline characteristics of the 4 subjects 1-5 months after optimization of the treatment of the underlying diseases and before the start of bosentan. At this point the $\mathrm{x}$-rays ruled out pneumonia, and no subject showed hypersecretion. There was no evidence of exacerbation, and no further modification of COPD treatment was required. The 4 subjects were of older age. While 2 of them showed severe airway obstruction, one showed moderate to severe airway obstruction, and one subject showed moderate airway obstruction. In 2 subjects the diffusion capacity for carbon monoxide was severely reduced, and those subjects were on long-term oxygen supplementation. All subjects were classified in World Health Organization functional class 3 . In 2 subjects 6-min walk distance (6MWD) was severely reduced. Three subjects had concomitant coronary artery disease, and one subject had a history of aortic valve replacement. Two subjects had severe $\mathrm{PH}$, while the other 2 had moderate $\mathrm{PH}$. One subject showed a pulmonary wedge pressure of $22 \mathrm{~mm} \mathrm{Hg}$, but all subjects had a transpulmonary vascular pressure difference of at least $23 \mathrm{~mm} \mathrm{Hg}$.

After starting bosentan, at the follow-up visits we measured World Health Organization functional class, 6MWD, echocardiography, electrocardiography, body plethysmography, blood gas analysis, and serum amino-terminal probrain natriuretic peptide (NT-proBNP) 2-6 months, for an overall follow-up period of 9-18 months. Right heart catheterization follow-up was performed once a year.

All the subjects improved with bosentan. There was an improvement of 6MWD in all subjects (Fig. 1). The maximum improvements were at 9, 13, and 18 months, respectively. The mean maximal 6MWD gain of all 4 subjects during the complete follow-up period was $142.5 \mathrm{~m}$. We compared the 6MWD gain of 3 subjects at 2 different time points. The $6 \mathrm{MWD}$ gain was $36 \mathrm{~m}$ after an early time point of 2 or 3 months, and $145 \mathrm{~m}$ after a later time point of 8 or 9 months. There was an improvement in tricuspid annular plane systolic excursion measured by echocardiography (Fig. 2), as well as improvement in mean pulmonary artery (Fig. 3). Oxygenation was stable (Fig. 4).

\section{Discussion}

We report on 4 subjects with COPD and PH. Three of them had coronary artery disease and atrial fibrillation, which are common comorbidities in COPD patients. Scharf et $\mathrm{al}^{6}$ reported that diastolic dysfunction is a frequent and relevant finding of patients with emphysema. In our subjects, coronary artery disease and atrial fibrillation may suggest pulmonary venous hypertension. One of the 4 subjects showed pulmonary artery wedge pressure
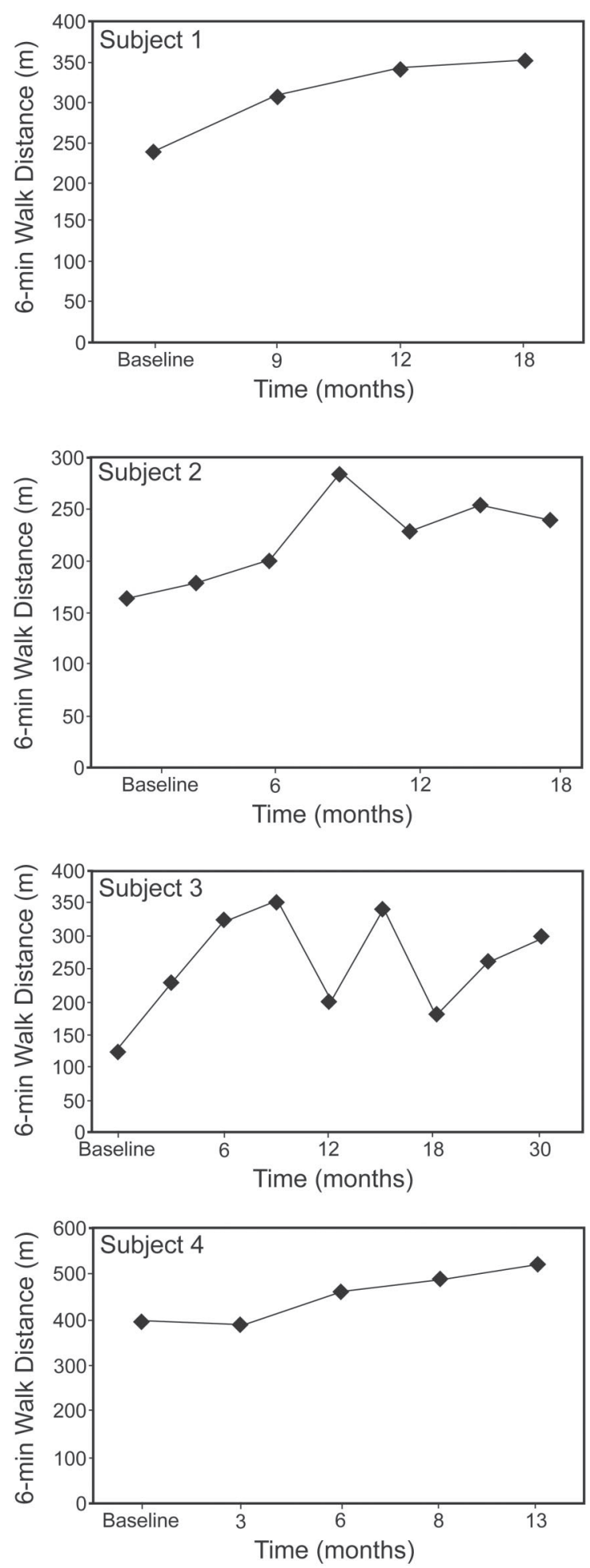

Fig. 1. Six-min walk distance of the 4 subjects.

$>15 \mathrm{~mm} \mathrm{Hg}$. The other 3 subjects showed a pulmonary artery wedge pressure of $12-15 \mathrm{~mm} \mathrm{Hg}$, characteristic of precapillary $\mathrm{PH}$ according to the guidelines ${ }^{8,9}$ and the Dana Point statement. In addition, acute vascular responsiveness 


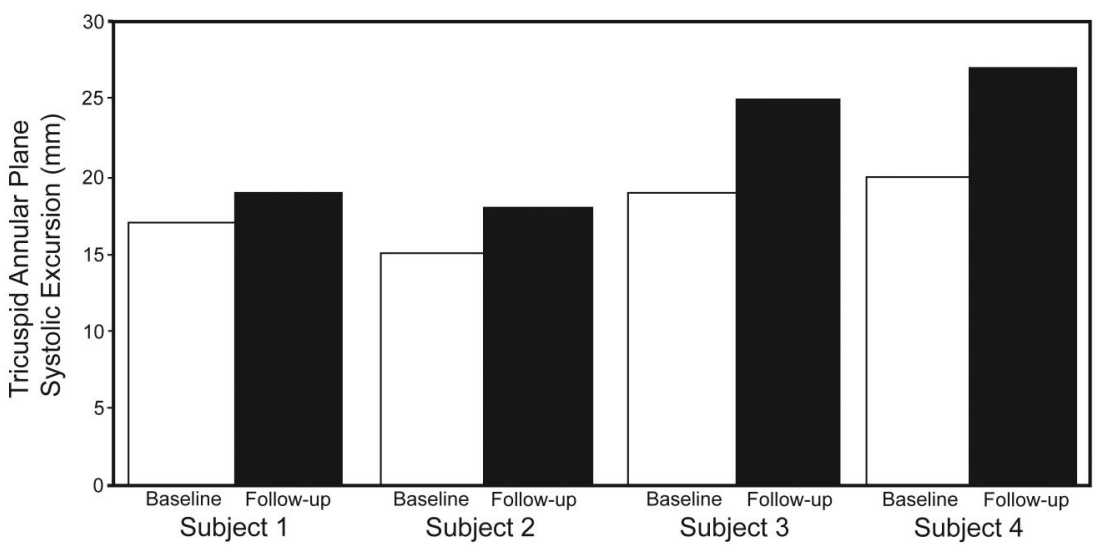

Fig. 2. Tricuspid annular plane systolic excursion at baseline and after 9 months of bosentan.

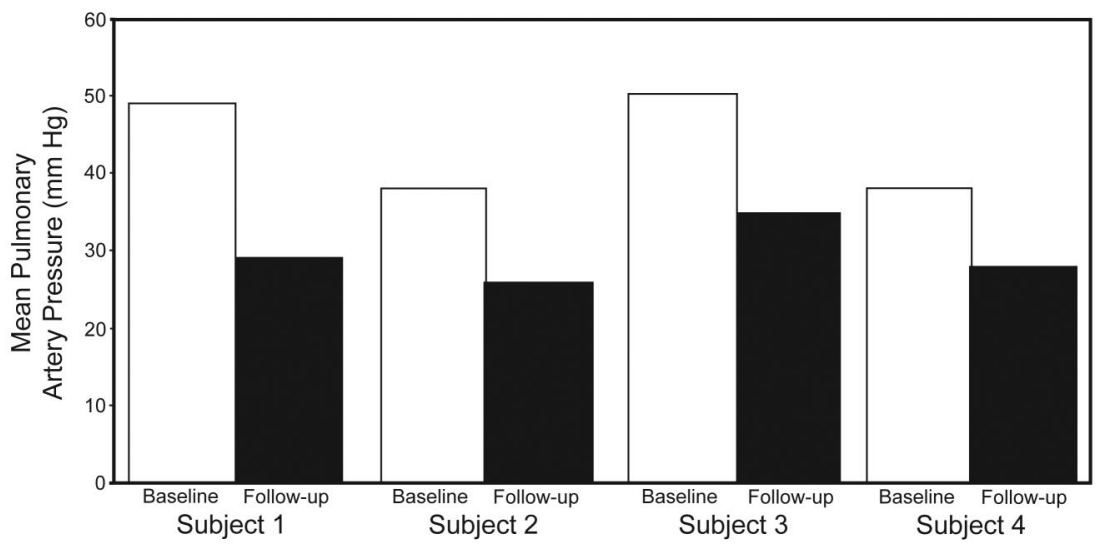

Fig. 3. Mean pulmonary artery pressure at baseline and at follow-up. After starting bosentan, right heart catheterization was done at 18 months in subjects 1 and 2, at 9 months in subject 3 , and at 8 months subject 4 .

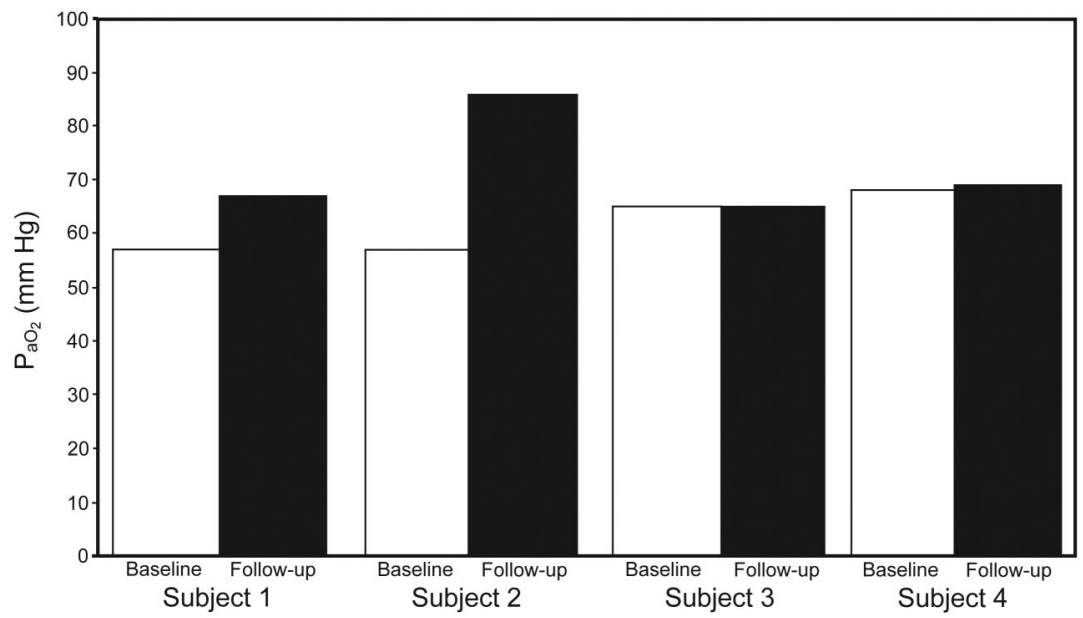

Fig. 4. $\mathrm{P}_{\mathrm{aO}}$ at baseline and after starting bosentan therapy, at 6 months in subject 1 , at 2 months in subject 2 , and at 3 months in subject 3 and 4.

was tested in Subject 1. Under inhaled iloprost, the mean pulmonary artery pressure improved from $44 \mathrm{~mm} \mathrm{Hg}$ to $28 \mathrm{~mm} \mathrm{Hg}$. Furthermore, it is questionable whether in
COPD patients, elevated pulmonary artery wedge pressure exclusively reflects diastolic left ventricular dysfunction. Air trapping and hyperinflation may increase intrathoracic 
pressure and, consequently, pulmonary artery pressure and pulmonary capillary wedge pressure. This could be characterized by simultaneously measuring the esophageal pressure, which was not done in the clinical setting. Since transpulmonary vascular difference was $23-28 \mathrm{~mm} \mathrm{Hg}$ in all the 4 subjects, we assumed a predominantly precapillary $\mathrm{PH}$. To uncover latent pulmonary venous hypertension, a "fluid challenge" procedure, which was not done in our subjects, should be performed in patients with cardiac comorbidities, administering a rapid bolus of $500 \mathrm{~mL} \mathrm{NaCl}$ and measuring pulmonary artery wedge pressure again.

Concomitant pulmonary arterial hypertension associated with collagen vascular disease and even chronic thromboembolic PH has been reported in COPD patients. ${ }^{4}$ In our subjects those conditions were carefully ruled out.

Three of our subjects showed severe airway obstruction. Only one subject showed criteria for the concept of "out of proportion PH" (ie, severe PH with only mild airway obstruction). For patients with PH and lung disease the current guidelines recommend treatment of the underlying disease. ${ }^{8,9}$ In our subjects, treatment of COPD and the other concomitant diseases had been optimized 1-5 months before specific treatment of $\mathrm{PH}$ was considered. Despite optimized treatment, severe $\mathrm{PH}$ persisted in 2 subjects, while moderate $\mathrm{PH}$ remained unchanged in the other 2.

All 4 subjects showed improvement following vasoactive treatment with bosentan. It is well known that in COPD patients, pulmonary artery pressure rises during exacerbation. ${ }^{15}$ In our subjects there were no clinical signs of COPD exacerbation, according to the GOLD criteria, ${ }^{14}$ when bosentan treatment was started. Although it is conceivable that hemodynamic and functional improvement was influenced by the optimization of the treatment of the underlying COPD by modifying anti-obstructive therapy, start of long-term oxygen therapy, and treatment of concomitant coronary artery disease by angioplasty 1-5 months prior to initiation of bosentan therapy, this seems unlikely. Since bosentan was started 1-5 months after these modifications, the hemodynamic and clinical improvement of $\mathrm{PH}$ might be the consequence of starting bosentan. The presence of a pulmonary vasculopathy in COPD with concomitant PH has been postulated by other authors. ${ }^{3,4,7}$

The positive effect of the endothelin receptor antagonist bosentan on PH due to hypoxic PH and COPD was shown in animal models as well as in humans. ${ }^{10-12}$ Stolz et al ${ }^{13}$ concluded that bosentan failed to improve functional capacity in COPD patients without severe $\mathrm{PH}$, but this cohort was not investigated by right heart catheterization, and the group treated with bosentan had a mean systolic pulmonary artery pressure of only $32 \mathrm{~mm} \mathrm{Hg}$.

In our subjects the 6MWD as a marker of clinical performance improved even more after a longer follow-up period: 3 subjects showed their maximum improvement after 9 months, and the remaining subject after 18 months.
The gain of 6MWD after 8 and 9 months was higher, compared to the second and third month. Studies evaluating the benefit of PH therapy have mostly had a follow-up of 12-16 weeks, which might be too short for assessing treatment effects.

Due to increasing shunt perfusion, pulmonary vasodilatation could lead to worsening of oxygenation in COPD patients. ${ }^{1,16}$ In our subjects, oxygenation remained stable and the treatment was safe.

This small retrospective case series cannot prove that the clinical and hemodynamic improvement in our subjects was due to bosentan treatment. We suggest that in future prospective clinical trials a longer follow up-period could be useful to investigate the true effect of pharmacologic treatment of PH in COPD.

To identify COPD patients who could possibly benefit from specific pharmacologic $\mathrm{PH}$ treatment, prospective clinical trials are needed. Patients should be carefully selected for inclusion in these trials. A follow-up period of only 16 weeks might lead to an underestimation of the treatment effects.

\section{REFERENCES}

1. Barberà JA, Blanco I. Pulmonary hypertension in patients with chronic obstructive pulmonary disease, advances in pathophysiology and management. Drugs 2009;69(9):1153-1171.

2. Thabut G, Dauriat G, Stern JB, Logeart D, Lévy A, Marrash-Chahla $\mathrm{R}$, Mal H. Pulmonary hemodynamics in advanced COPD candidates for lung volume reduction surgery or lung transplantation. Chest 2005;127(5):1531-1536.

3. Chaouat A, Naeije R, Weitzenblum E. Pulmonary hypertension in COPD. Eur Respir J 2008;32(5):1371-1385.

4. Chaouat A, Bugnet AS, Kadaoui N, Schott R, Enache I, Ducoloné A, et al. Severe pulmonary hypertension and chronic obstructive pulmonary disease. Am J Respir Crit Care Med 2005;172(2):189-194.

5. Oswald-Mammoser M, Weitzenblum E, Quoix E, Moser G, Chaouat A, Charpentier C, Kessler R. Prognostic factors in COPD patients receiving long-term oxygen therapy: importance of pulmonary artery pressure. Chest 1995;107(5):1193-1198.

6. Scharf SM, Iqbal M, Keller C, Criner G, Lee S, Fessler HE; National Emphysema Treatment Trial (NETT) Research Group. Hemodynamic characterization of patients with severe emphysema. Am J Respir Crit Care Med 2002;166(3):314-322.

7. Hale K, Niewoehner D, Cosio MG. Morphologic changes in the muscular pulmonary arteries: relationship to cigarette smoking, airway disease, and emphysema. Am Rev Respir Dis 1980;122(2): 273-278.

8. Galiè N, Hoeper MM, Humbert M, Torbicki A, Vachiery JL, Barberà JA, et al; Task Force for the Diagnosis and Treatment of Pulmonary Hypertension of the European Society of Cardiology (ESC) and the European Respiratory Society (ERS) endorsed by the International Society of Heart and Lung Transplantation (ISHLT). Guidelines for the diagnosis and treatment of pulmonary hypertension. Eur Respir J 2009;34(6):1219-1263.

9. Hoeper MM, Andreas S, Bastian A, Claussen M, Ghofrani HA, Gorenflo M, et al. Pulmonary hypertension due to chronic lung disease: updated recommendations of the Cologne Consensus Conference 2011. Int J Cardiol 2011;154(Suppl 1):S45-S53. 


\section{PULMONARY HyPERTENSION IN COPD}

10. Chen SJ, Chen YF, Meng QC, Durand J, Dicarlo VS, Oparil S. Endothelin-receptor antagonist bosentan prevents and reverses hypoxic pulmonary hypertension in rats. J Appl Physiol 1995;79(6): 2122-2131.

11. Roig Figueroa V, Herrero Perzez A, Abad Lecha E, Ramos Cancelo I, Vielba Ramos D. Bosentan in pulmonary hypertension associated with hypoxaemic lung diseases. Curr Med Res Opin 2007;23(Suppl 2):S49-S53

12. Valerio G, Bracciale P, Grazia D'Agostino AG. Effect of bosentan upon pulmonary hypertension in chronic obstructive pulmonary disease. Ther Adv Respir Disease 2009;3(1):15-21.

13. Stolz D, Rasch H, Linka A, Di Valentino M, Meyer A, Brutsche M, Tamm M. A randomised, controlled trial of bosentan in severe COPD. Eur Respir J 2008;32(3):619-628.
14. Rabe KF, Hurd S, Anzueto A, Barnes PJ, Buist SA, Calverley P, et al; Global Initiative for Chronic Obstructive Lung Disease. Global strategy for the diagnosis, management, and prevention of chronic obstructive pulmonary disease: GOLD executive summary. Am J Respir Crit Care Med 2007;176(6):532-555.

15. Abraham AS, Cole RB, Green ID, Hedworth-Whitty RB, Clarke SW, Bishop JM. Factors contributing to the reversible pulmonary hypertension of patients with acute respiratory failure studied by serial observations during recovery. Circ Res 1969;24(1):51-60.

16. Blanco I, Gimeno E, Muñoz PA, Pizarro S, Gistau C, RodriguezRoisin R, et al. Hemodynamic and gas exchange effects of sildenafil in patients with chronic obstructive pulmonary disease and pulmonary hypertension. Am J Respir Crit Care Med 2010;181(3): 270-278. 